\title{
Academia and industry united
}

\author{
A joint research platform is a great playground for young researchers to combine fundamental and \\ applied research, says Diana Nanova.
}

tech have always been fascinated by technological progress and this was the main reason why I decided to study physics. Over the course of my studies, I developed an interest in nanostructures and when I decided to study for my $\mathrm{PhD}$ I wanted to combine my original interest in innovative applications with my more recent interests. The InnovationLab in Heidelberg, Germany, promised to be a good option because it is a joint multidisciplinary research platform for academia and industry. It focuses on printed and organic electronics and ultimately aims to link fundamental research to real-life applications. Working at InnovationLab meant that although I would officially be a student of the Heidelberg University doing fundamental research, I would also be involved in projects with partners from the chemical and the solar power industries.

Organic semiconductors present fundamental challenges but also technological opportunities. In terms of applications they can enable low-cost printing processes for light-emitting diodes, solar cells or sensors. Along with their lightweight, flexible and transparent properties, organic semiconductors allow innovative applications such as the integration of solar cells in building facades, rollable TV screens or flexible pressure sensors, to name just a few. From a fundamental perspective, organic semiconductors are mostly amorphous and suffer from a lack of long-range order. The way this structural disorder influences key properties, such as conductivity, which is important for the function of semiconducting devices, is not completely understood, making fundamental research necessary. There is obviously much to explore in both the fundamental and applied aspects of this field, so why not combine both approaches in joint projects?

As part of my PhD at the InnovationLab, I studied the complex relationship between the structural and electronic properties of organic semiconductors, in particular those used in solar cells. To investigate the structure of the semiconductor, I

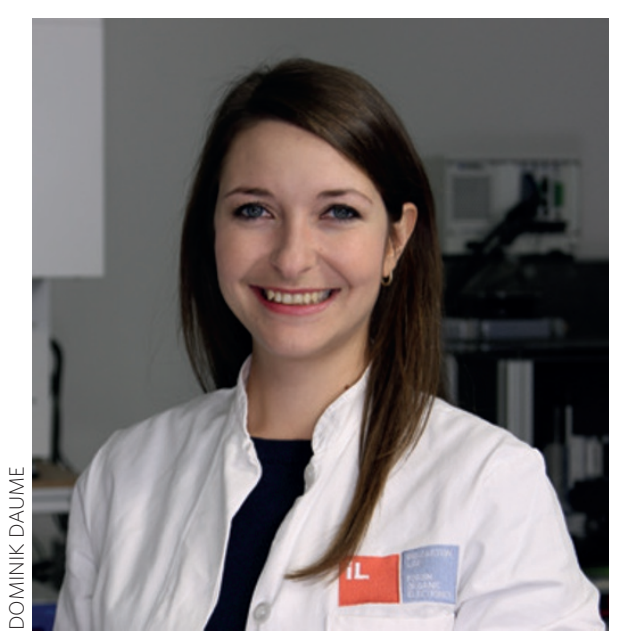

sophisticated TEM measurements become even more interesting if you apply them to real devices and real devices are more useful if you understand how they work.

Of course, there were also some problems with this collaboration. The downsides of working with a big industrial company, for example in terms of confidentiality and information exchange, can be frustrating for young researchers who want to publish their results. Although it didn't affect me strongly, because I also worked on non-confidential projects in parallel, I believe that this could potentially be a big hindrance for other young researchers who depend on numerous publications to excel in a competitive environment. Additionally, some of the research is about optimizing process parameters for the best-performing devices and this can be disappointing if you are more interested in understanding the reasons why devices do or do not work. Overall, however, I had the chance to learn a different approach to research and I acquired some knowledge on how industry research is evolving and which technologies are considered to be state of the art.

The joint research platform was a great opportunity to experience research from different perspectives. Interaction with industry and the other groups taught me how to place my research in a greater context and to see the big picture. The insights into company research are exciting and can be a competitive advantage for a future career, especially in an applicationoriented field such as organic electronics. Along with the large network in academia and industry, the joint multidisciplinary research platform, in principle, opens doors to any career path. The majority of $\mathrm{PhD}$ students that graduate, however, eventually choose a way out of academia. Maybe it is due to the research field or the future prospects - or is it just a coincidence?

DIANA NANOVA recently completed her $\mathrm{PhD}$ in physics at the InnovationLab, Heidelberg University, Speyerer Str. 4, 69115 Heidelberg, Germany. e-mail: diana.nanova@gmail.com 